\title{
Dosing enzyme replacement therapy for Gaucher disease: older, but are we wiser?
}

\author{
Ellen Sidransky, $M D^{1}$, Gregory M. Pastores, $M D^{2}$, and Motomi Mori, $P h D^{3}$
}

$\mathrm{E}_{\mathrm{a}}^{\mathrm{n}}$ nzyme replacement for Gaucher disease (GD) has now been available for 17 years. This therapy, the first enzyme preparation developed for the treatment of a lysosomal storage disorder, has dramatically changed the natural history of type 1 GD. However, while the therapy is now widely used around the world, there still is not a consensus regarding dosing recommendations or even indications for initiating therapy. Grabowski and colleagues attempt to address this issue once and for all in their article, using a database of clinical data assembled by the International Collaborative Gaucher Group on 4434 patients to establish dose-response relationships, focusing on doses and disease parameters used in clinical practice. Unfortunately, despite all of their painstaking efforts, uncertainty remains concerning optimal management for this disorder.

Why is this important? After all, an efficacious drug is available, and patients are doing remarkably better, with dramatically fewer splenectomies, transfusions, surgeries, and bleeds. If high doses are successful, without significant adverse effects, why is there so much concern? In this case, it is all about cost. Imiglucerase (Cerezyme $\AA$ ) is among the most expensive drugs on the market, costing approximately $\$ 5.50 /$ unit. The doses evaluated in this study ranged from 5 to $75 \mathrm{U} / \mathrm{kg}$ every other week, which corresponds to an annual expenditure of $\$ 43,000$ versus $\$ 642,900$ in drug cost alone for a $60 \mathrm{~kg}$ adult.

Ideally, as stated by the authors, dose-response relationships should be evaluated by clinical trials. Because of ethical, commercial, and feasibility issues, however, this has not been possible and the authors maintain that analysis of data from the International Collaborative Gaucher Group provides a suitable alternate means to approximate a randomized trial. Specifically, they applied a propensity score matching method to create a pseudorandomized trial, creating groups that were similar with respect to age and baseline clinical characteristics. They should be commended for undertaking this challenge, which required enormous effort, including logistic regression analyses and nonlinear mixed effects modeling. Based on their analysis, they concluded that the study provided strong evidence of incremen-

From the ${ }^{1}$ Medical Genetics Branch, National Human Genome Research Institute, National Institutes of Health, Bethesda, Maryland; ${ }^{2}$ Departments of Neurology and Pediatrics, New York University School of Medicine, New York, New York; and ${ }^{3}$ OHSU Cancer Institute and Oregon Clinical and Translational Research Institute Oregon Health \& Science University, Portland, Oregon.

Ellen Sidransky, MD, Section on Molecular Neurogenetics, National Human Genome Research Institute, Building 35, Room 1A213, 35 Convent Drive, MSC 3708, Bethesda, MD 20892-3708. E-mail: sidranse@mail.nih.gov

Ellen Sidransky and Motomi Mori declare no conflict of interest.

Gregory M. Pastores is the recipient of research grants from Actelion, Amicus, Biomarin, Genzyme, Protalix, and Shire HGT; pharmaceutical/biotechnology companies engaged in the development of therapies for the lysosomal storage disorders.

Submitted for publication October 9, 2008.

Accepted for publication October 14, 2008.

DOI: $10.1097 /$ GIM.0b013e3181928f6a tal dose-response relationships, with a more robust response seen with high dose therapy at $60 \mathrm{IU} / \mathrm{kg}$ every other week.

On the surface, this seems straightforward. The authors evaluated four clinical parameters; hemoglobin concentration, platelet count, and hepatic and splenic volumes, and showed that for three, the maximal effect of dose was statistically significantly higher for subjects treated with the highest doses. However, the more immediate question is whether these statistical differences have clinical relevance. For example, after 1 year of therapy, all three dosage groups demonstrated an improvement in hemoglobin levels of at least $1 \mathrm{~g} / \mathrm{dL}$. Given that the mean baseline hemoglobin level for each group was reported as being within the normal range, is a still higher hemoglobin level a reflection of a better response? Likewise, the rise in platelet counts was difficult to interpret clinically. Is a platelet count of $110 \mathrm{~K}$ functionally different from $120 \mathrm{~K}$ ? Remarkably, by the end of the period evaluated, all three groups had mean platelet counts between $188 \mathrm{~K}$ and $198 \mathrm{~K}$. Finally, while the initial differences in the rate and degree of reduction of splenic volumes were more convincing, by 12 months, all three groups had marked and similar reductions.

The authors state that "in the absence of a validated outcome score and consensus agreement on the appropriate clinical responses, it is not yet possible to provide general clinical guidelines." By making this statement they totally skirt the issue of clinical efficacy. Yet they state that the "outcomes of the study will certainly be of value when considering individual choices for treatment dose." If the results do not have applicability that can be generalized, how can they be used for managing individual patients?

One reason why the reader does not become a great deal wiser from this study is that the data can easily be used to support each reader's preconceived view on this issue. Those that argue for high dose will feel that the study confirms that higher doses result in greater efficacy. Those who advocate for low doses will argue that an acceptable response was achieved with the lower doses at a far lower cost. Others who recommend mid-range doses will feel satisfied that their strategy balances cost and efficacy. Also, while it remains possible that there are individuals in each group that require higher doses for optimal response, the article does not provide guidance on how to identify such patients.

Perhaps the most remarkable result of the study is that all three groups of patients, regardless of the dosing regimen used, demonstrated improvements, with all clinical parameters returning to within the normal range. One could argue from this composite data that each of the doses tested is acceptable, and that the dose selected is less critical than generally believed. If that is indeed the case, then cost considerations become extremely important. For a $60 \mathrm{~kg}$ adult, the saving in drug cost for those receiving a $15 \mathrm{U} / \mathrm{kg}$ dose versus a $60 \mathrm{U} / \mathrm{kg}$ dose amounts to more than half a million dollars per year.

Thus, how does this study help the community treating Gaucher disease? The authors refer to the 1995 NIH Technology Assessment conference on the Diagnosis and Treatment of Gaucher disease, in which the panel highlighted the importance 
of determining "the lowest effective dosage for clinical response." Although the authors interpret their data as showing that "higher dose groups demonstrated greater treatment effects," it could be argued that the lowest dosage group studied also demonstrated an effective clinical response at a far lower cost, at least in the groups selected for comparison.

Another potential concern with this study is the fact that the biostatisticians participating were employed by Genzyme Corporation, and the patient database used is funded and coordinated by the pharmaceutical company with the most to gain from the use of higher doses. The NIH panel specifically recommended that any such database be "independent of any corporate entity, particularly those involved in the screening, diagnosis, management, or treatment of Gaucher disease," cautioning against the current dilemma. Although the authors include distinguished leaders in the field of GD, and existing conflicts of interest have been appropriately disclosed by the authors, there remains the difficulty of relying on data collected and analyzed by a company so invested in the results. Yet many have argued that the patient registry only exists because of Genzyme Corporation's willingness to generously fund it. This issue has great relevance for other rare disorders. Granting authorities like the NIH should appreciate the necessity of funding registries and sample repositories for rare diseases, so that there is not a need for corporate entities to fill the void.

So with 17 years of experience and this plethora of data on a rare disease, why do we not feel wiser? Perhaps because for those treating GD, there are other crucial issues that have not been satisfactorily addressed. These include the criteria for initiating treatment, frequency of dosing, when dosing can be tapered or stopped, and whether pediatric and adult dosing should both be on a per kilogram basis. This study did not consider the effect of the drug on bony manifestations, a considerable cause of morbidity in this patient group. Furthermore, other strategies to improve the drug's half life and administration should be explored. Can complementary strategies be formulated to prevent a life-long reliance on enzyme infusions? Because this study suggests that the issue of dose is not as critical as presumed, it is time for the community to come together to attack some of the other remaining relevant issues pertaining to the treatment of GD, which serves as the prototype for the treatment of many other rare disorders.

\section{ACKNOWLEDGMENTS}

Ellen Sidransky's contribution to this work was funded by the Intramural Research Program of the National Human Genome Research Institute, National Institutes of Health. 